ARTICLE

DOI: 10.1038/s41467-017-01812-w OPEN

\title{
Benchmarking organic mixed conductors for transistors
}

Sahika Inal (1) 1, George G. Malliaras (1) ${ }^{2,5}$ \& Jonathan Rivnay (i]) ${ }^{3,4}$

Organic mixed conductors have garnered significant attention in applications from bioelectronics to energy storage/generation. Their implementation in organic transistors has led to enhanced biosensing, neuromorphic function, and specialized circuits. While a narrow class of conducting polymers continues to excel in these new applications, materials design efforts have accelerated as researchers target new functionality, processability, and improved performance/stability. Materials for organic electrochemical transistors (OECTs) require both efficient electronic transport and facile ion injection in order to sustain high capacity. In this work, we show that the product of the electronic mobility and volumetric charge storage capacity $\left(\mu C^{\star}\right)$ is the materials/system figure of merit; we use this framework to benchmark and compare the steady-state OECT performance of ten previously reported materials. This product can be independently verified and decoupled to guide materials design and processing. OECTs can therefore be used as a tool for understanding and designing new organic mixed conductors.

\footnotetext{
${ }_{1}^{1}$ Biological and Environmental Sciences and Engineering Division, King Abdullah University of Science and Technology (KAUST), Thuwal 23955-6900, Saud Arabia. ${ }^{2}$ Department of Bioelectronics, Ecole Nationale Supérieure des Mines, CMP-EMSE, MOC, 13541 Gardanne, France. ${ }^{3}$ Department of Biomedical Engineering, Northwestern University, Evanston, IL 60208, USA. ${ }^{4}$ Simpson Querrey Institute for BioNanotechnology, Northwestern University, Chicago, IL 60611, USA. ${ }^{5}$ Present address: Electrical Engineering Division, Department of Engineering, University of Cambridge, 9 JJ Thomson Avenue, Cambridge CB3 OFA, UK. Correspondence and requests for materials should be addressed to J.R. (email: jrivnay@northwestern.edu)
} 
O rganic mixed conductors are soft, often polymeric materials based on traditional organic electronic materials. These materials support electronic charge transport along their conjugated backbones, while allowing for ionic (mass) transport through the bulk. Like their inorganic counterparts, it is the interaction of ionic and electronic species that allow for the diverse applications ranging from battery electrodes to electrochromic windows and sensors ${ }^{1}$. The interplay between ionic and electronic carriers is critical for energy storage and generation including pseudo/supercapacitors ${ }^{2,} 3$, batteries ${ }^{4}$, fuel cells $^{5}$ and ionic-organic ratchets ${ }^{6}$. Furthermore, organic mixed conductors have found utility in applications where large area processing is required (i.e., electrochromics), where the motion of ions through the bulk can lead to significant changes in physical properties (mechanical actuators, electrochromics, switchable surfaces) ${ }^{7-9}$, or where the material's intimate contact with a sensing environment can lead to enhanced sensitivity or selectivity (sensors) ${ }^{10,11}$. Where one application requires selectivity, another requires fast response; where one requires stability, another requires capacity. While device-based characterization of such properties enables comparison, lacking is a materials-based figure of merit to benchmark and guide synthetic/processing design and development.

Organic electrochemical transistors (OECTs) have received significant attention for their promise as circuit elements ${ }^{12}$, neuromorphic devices ${ }^{13}$, and sensing/stimulation elements useful in bioelectronics applications ${ }^{13-15}$. The ability for organic mixed conductors to support ion penetration (and thus bulk charge capacity) has allowed for high performance OECTs to be developed as amplifying transducers for bioelectronic applications ${ }^{15}$. In many cases, the enhanced sensing capability of OECTs has allowed them to outpace both mixed conductor-coated electrodes and similarly sized inorganic transistors based on non-mixed conductors 16,17

In an OECT, the mixed conductor channel bridging a source and drain electrode is in direct contact with an electrolyte rich in mobile ions (Fig. 1). The current between source and drain electrodes, $I_{\mathrm{D}}$, is modulated by a change in effective gate bias, $V_{G}$ (applied at an immersed gate electrode or resulting from cellular or (bio)molecular activity), through a bulk gating effect facilitated by ion penetration. Operation through bulk doping yields both low bias operation, a hallmark of electrolyte gated transistors, but also high capacitance. Due to the motion of both electronic and ionic charges in the channel, mobility extraction cannot be, in a straight forward manner, performed via the transconductance method as applied for field effect transistors (FETs). For this reason, OECT researchers often report device transconductance, which is the slope of the $I_{\mathrm{D}}-V_{\mathrm{G}}$ transfer curve, $g_{\mathrm{m}}=\partial I_{\mathrm{D}} / \partial V_{\mathrm{G}}$ (see Fig. 1b). While this term is sometimes reported as a normalized value (e.g., by channel width, $W$, or by drain voltage, $V_{\mathrm{D}}$ ), reporting standards are not well established. Nevertheless, $g_{m}$ is a direct measure of effective signal amplification of a single OECT; which, for instance, determines whether the OECT will be able to operate as a biosensor, i.e., transduce small biological signals. Furthermore, $g_{\mathrm{m}}$ depends on both the channel geometry and biasing conditions and describes the steady-state performance of the device. As such, it does not represent the performance of the mixed conductor, yet can be used to derive an appropriate figure of merit for fair materials comparison.

The conducting polymer poly(3,4-ethylenedioxythiophene) complexed with poly(styrene sulfonate) (PEDOT:PSS) is among the most commonly studied materials in OECT research, and has allowed for significant device physics and modeling development. It is stable in aqueous conditions, processable by a variety of methods, allows for both electronic and ionic transport, and importantly, is readily commercially available ${ }^{3,18}$. Previous work has shown that volumetric capacitance $\left(\sim 40 \mathrm{~F} \mathrm{~cm}^{-3}\right.$ in PEDOT: PSS) governs high transconductance; consequently, the physical film thickness of the OECT channel, $d$, plays a critical role in determining both transient and steady-state device characteristics $^{15}$. These findings can be corroborated and derived ${ }^{15}$, resulting in the following scaling of OECT transconductance:

$$
g_{\mathrm{m}}=\frac{W d}{L} \mu C^{*}\left(V_{\mathrm{th}}-V_{\mathrm{G}}\right)
$$

Where $\mu$ is the electronic carrier mobility, and $C^{*}$ is the capacitance of the channel per unit volume. $V_{\text {th }}$ is the threshold voltage. In the limiting case where the channel thickness drops to a monolayer, the product of $d$ and $C^{*}$ is $C^{\prime}$ (capacitance per unit area), which collapses to the transconductance equation for FETs:

$$
g_{\mathrm{m}}=\frac{W}{L} \mu C^{\prime}\left(V_{\mathrm{th}}-V_{\mathrm{G}}\right)
$$

In the equation governing the OECT performance (Eq. (1)), the geometry dependence is captured by the $W d / L$ term and the gate bias offset by $\left(V_{\text {th }}-V_{\mathrm{G}}\right)$. This leaves the product of $\mu$ and $C^{\star}$ as the only nominally geometry- and bias-independent terms, and thus a materials-system dependent product.

Device-based figures of merit that are treated as materials properties are especially useful in materials evaluation and development. For example, OFET materials are often benchmarked based on their $\mu_{\mathrm{FET}}$, while thermoelectric materials are
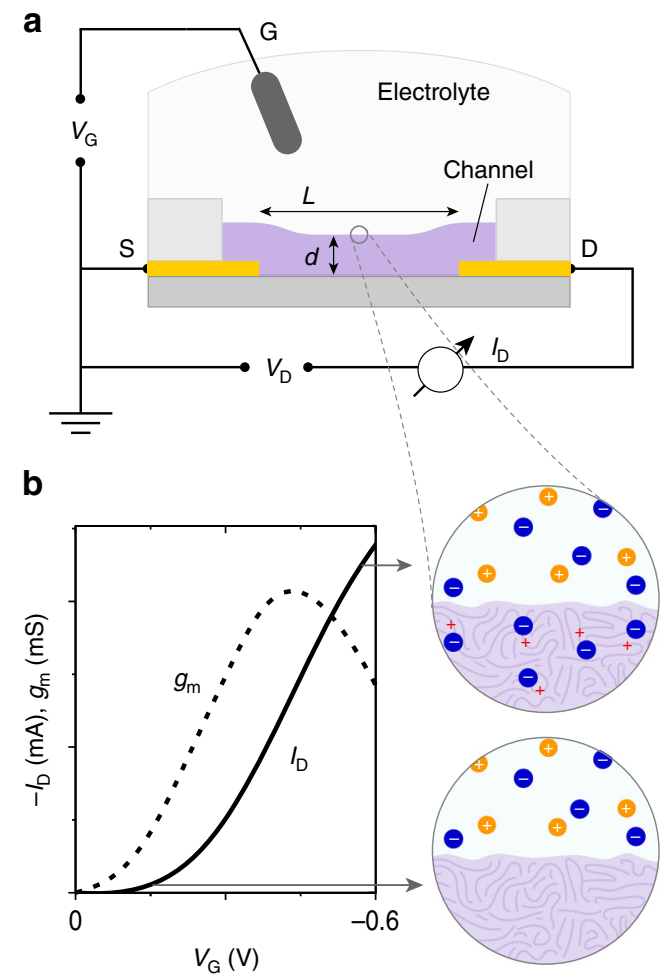

Fig. 1 OECT description and operation. a OECT cross section, wiring, and dimensions: channel length $(L)$, and thickness $(d)$. The source, drain, and gate $\left(S, D, G\right.$, respectively), and relevant voltages (gate, drain voltage: $V_{G}$ $V_{D}$ ) and currents (drain current, $I_{D}$ ) terms are also labeled. $\mathbf{b}$

Representative transfer $\left(I_{D}-V_{G}\right)$ curve for a p-type accumulation mode device $\left(V_{D}<0 V\right)$, and the corresponding transconductance curve $\left(g_{m}\right)$. The schematics on the right indicate the doping state of the film, where the "ON" state allows for anion drift/penetration and subsequent stabilization of holes on the semiconducting backbone. In the schematics, cations are orange, anions are blue, and holes are red 

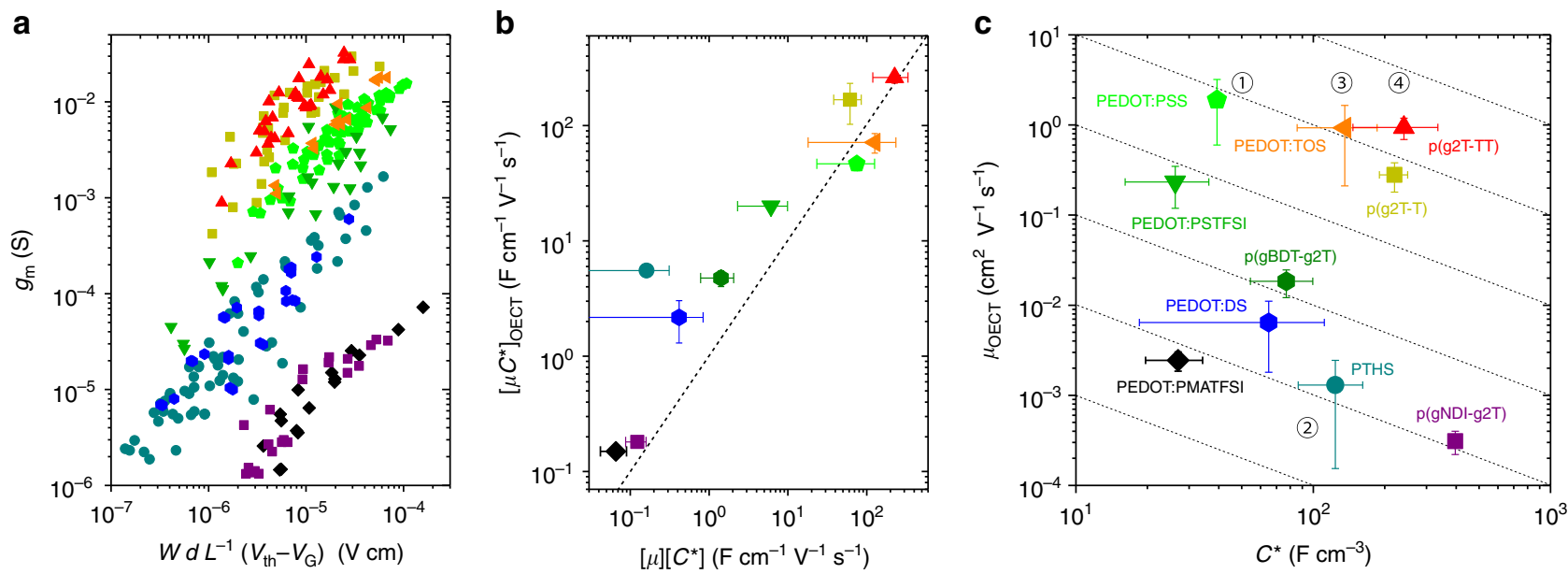

Fig. $2 \mu C^{\star}$ as the OECT figure of merit. a Transconductance $\left(g_{m}\right)$ of OECTs as a function operating conditions (in saturation regime) and channel geometry. Each point represents one OECT measurement; each color/shape represent one material, as indicated in the labeling in $\mathbf{c}$. $\mathbf{b}$ The linear slope of the data in $\mathbf{a}$, $\left[\mu C^{\star}\right]_{\mathrm{OECT}}$, as a function of the product of $\mu_{\mathrm{OECT}}$ and $C^{\star}$ determined independently, $[\mu]\left[C^{\star}\right]$. The dashed line represents 1:1 agreement between the values. Error bars represent the propogation of errors from the product of $\mu$ and $C^{\star}$ for which the standard deviation is determined over all devices tested for each material, as well as the error in the linear fit of data from each material in $\mathbf{a}$. The color coding and shape for each material is as noted in $\mathbf{c}$. $\mathbf{c} \mu_{\mathrm{OECT}}-\mathrm{C}^{\star}$ map of ten previously reported materials. Dotted lines denote constant $\mu \mathrm{C}^{\star}$ product. Circled numbers in $\mathbf{c}$ refer to the discussion in text

defined with their $Z T$ figure of merit. It is proposed herein that the $\mu C^{*}$ product is the materials-system figure of merit for OECTs. This term captures the steady-state ionic/electronic transport processes within the channel material, under device operation. Herein, we use this framework to compare and benchmark ten previously published organic mixed conductor materials. We further explore how a decoupling of $\mu C^{\star}$ into $\mu$ and $C^{\star}$ can aid in materials design, including synthetic strategies and processing approaches. Knowing the $\mu$ and $C^{*}$ of the channel material, we can predict the steady-state performance, i.e., $g_{\mathrm{m}}$, of an OECT given a certain geometry and operation condition. We suggest that the $\mu C^{*}$ product could be useful for informing materials selection in other mixed conductor applications.

\section{Results}

$\boldsymbol{\mu} \boldsymbol{C}^{\star}$ for benchmarking OECT materials. $\mu C^{\star}$ captures the mixed ionic-electronic transport properties of the OECT channel material. When determined using Eq. (1), $\mu$, the electronic mobility, reflects the device-scale electronic charge transport in the channel, and has been shown to be comparable to values determined by complementary methods, such as Hall effect mobility ${ }^{19}$. $C^{\star}$ is a measure of the capacity of the bulk material (a volumetric term, as opposed to gravimetric, often used for energy storage devices). This term is a convolution of both the ionic penetration/transport within the polymeric morphology, and the ability for the conjugated backbone to store and collect electronic charges. Ultimately, $C^{*}$ should be a steady-state characteristic, and should reach this value at experimentally or application relevant time scales.

To extract the $\mu C^{*}$ product, it is sufficient to measure $g_{\mathrm{m}}$ and carefully note the device geometry parameters and biasing conditions. However, a more rigorous investigation, where multiple devices of varying geometries are measured, allows for both proper verification of OECT scaling (i.e., $g_{\mathrm{m}} \sim W d / L$ ), and for a more statistically supported $\mu C^{\star}$ determination. Following Eq. (1), the data can be plotted as $g_{\mathrm{m}} v s$. $W d L^{-1}\left|V_{\text {th }}-V_{\mathrm{G}}\right|$, where the slope is $\left[\mu C^{*}\right]_{\text {OECT. }}$. This is shown for the materials investigated in Fig. 2a. Variability or spreading in values can be attributed to non-uniformities in film formation, edge effects and their amplified effects in small devices, and difficulty in measuring thickness for small devices. This $\mu C^{*}$ product is then plotted for all ten materials in Fig. 2b, where a clear ranking of materials is evident.

Decoupling these terms can provide both validation and lead to a facile route to understand why one material outperforms another. For these materials, $\mu$ is determined by extracting the electronic carrier transit time $\left(\tau_{e}\right)$ through the constant gate current $^{20}$, or impedance matching methods previously reported ${ }^{21}$ and described in Methods. $C^{*}$ is measured independently by performing electrochemical impedance spectroscopy (EIS) measurements on a polymer coated microelectrode. The EIS results can be, in all materials discussed here, fit to a simple equivalent circuit: $R_{\mathrm{s}} C$ or $R_{\mathrm{s}}\left(R_{\mathrm{p}} \| C\right)$ where $R_{\mathrm{p}}$ and $C$ are the parallel resistance and the capacitance associated with the polymer, respectively, while $R_{\mathrm{s}}$ is the sum of the electrolyte and interconnect resistances (Supplementary Fig. 1). The capacitive term in the circuit is then normalized by the product of the measured electrode area and film thickness, i.e., the volume of the film. It is required that a material operates as a true OECT: at reported biases, ion penetration should progress through the bulk of the film (in this simplistic case, lateral ion transport under the applied drain bias is presently neglected)-meaning no stratification, and no dominance from ion accumulation at the electrolyte/channel interface (otherwise accurate $C^{\star}$ extraction is non-trivial, and the OECT operation dictated by Eq. (1) does not apply).

The product of these independently measured values $[\mu]$ and $\left[C^{*}\right]$ agree well with the $\left[\mu C^{*}\right]$ product attained from geometrydependent transconductance plot shown in Fig. 2a (Fig. 2b, Table 1). While many materials do not show a direct 1:1 match and often trend towards an underestimation in the independently derived product $[\mu]\left[C^{*}\right]$, the relative relation amongst different materials is maintained. The conjugated polyelectrolyte, PTHS, shows the most significant deviation. With this general agreement in trends, we can properly benchmark materials for OECTs. For example, the prototypical PEDOT:PSS exhibits a $\mu C^{\star}$ of $47 \pm 6 \mathrm{~F}$ $\mathrm{cm}^{-1} \mathrm{~V}^{-1} \mathrm{~s}^{-1}$. The top performer to date is $\mathrm{p}(\mathrm{g} 2 \mathrm{~T}-\mathrm{TT})$, a glycolated thiophene-thienothiophene polymer ${ }^{22}$, with a $\mu C^{*}$ of $261 \pm 29 \mathrm{~F} \mathrm{~cm}^{-1} \mathrm{~V}^{-1} \mathrm{~s}^{-1}$. It is important to note that in all of the materials shown in Fig. 2, testing was performed with the same setup, using a $\mathrm{Ag} / \mathrm{AgCl}$ pellet as the reference/gate electrode and $0.1 \mathrm{M} \mathrm{NaCl}$ as the electrolyte. 
Table 1 Materials figure of merit for various OECT materials

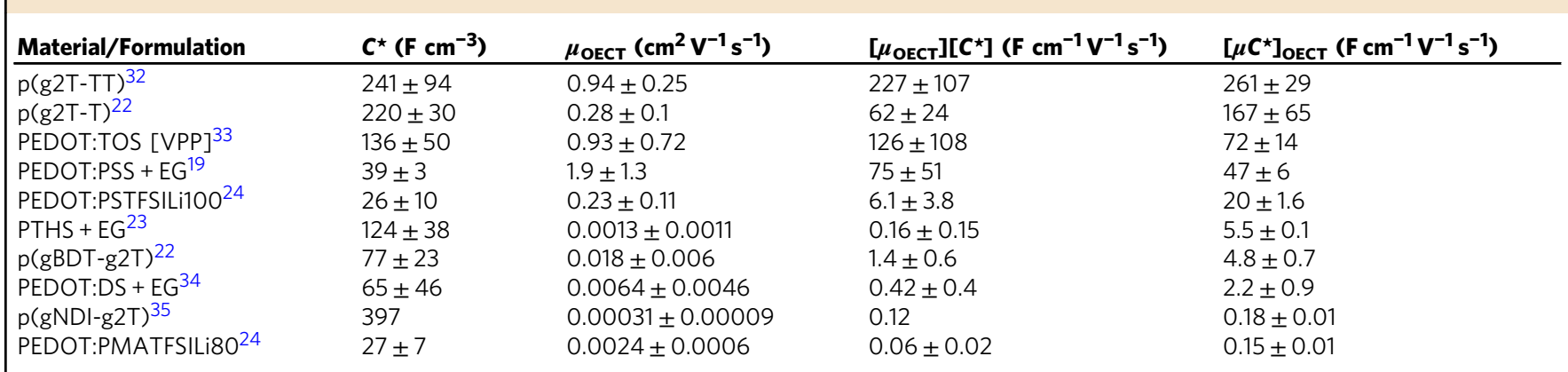

$\mathrm{p}(\mathrm{g} 2 \mathrm{~T}-\mathrm{T}), \mathrm{p}(\mathrm{g} 2 \mathrm{~T}-\mathrm{TT})$ and $\mathrm{p}(\mathrm{gBDT}-\mathrm{g} 2 \mathrm{~T})$ are glycolated thiophene, thiophene-thienothiophene and BDT-thiophene based polymers, respectively. TOS, PSS, PSTFSILi100 and PMATFSILi80, and DS, are various molecular, polymeric, and biological anionic dopants complexed with PEDOT, namely tosylate, poly(styrene sulfonate), (trifluoromethylsulfonyl)sulfonylimide (styrenic or methacrylic backbone, with a molar mass 100 or $80 \mathrm{kDa}$ and $\mathrm{Li}^{+}$as the counter ion), and dextran sulfate, respectively. $\mathrm{p}(\mathrm{gNDI}-\mathrm{g} 2 \mathrm{~T})$ is a glycolated naphthalene diimide based polymer, which is the only $\mathrm{n}$-type semiconductor in this work. PTHS is a thiophene-based conjugated polyelectrolyte with sulfonate terminated alkyl chains. Ethylene glycol (EG) is a co-solvent additive known to improve electronic conductivity of polymer films
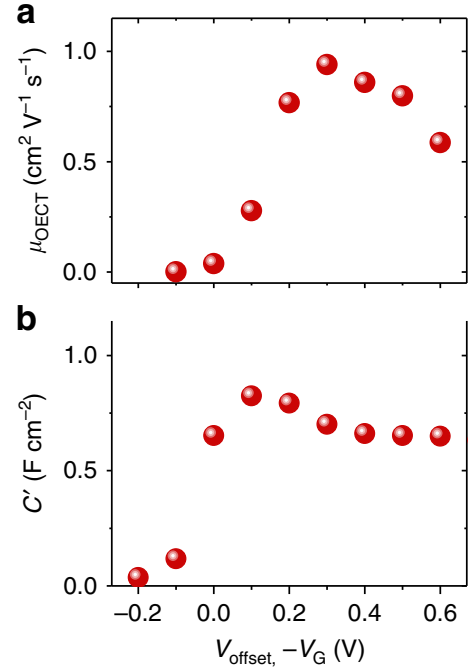

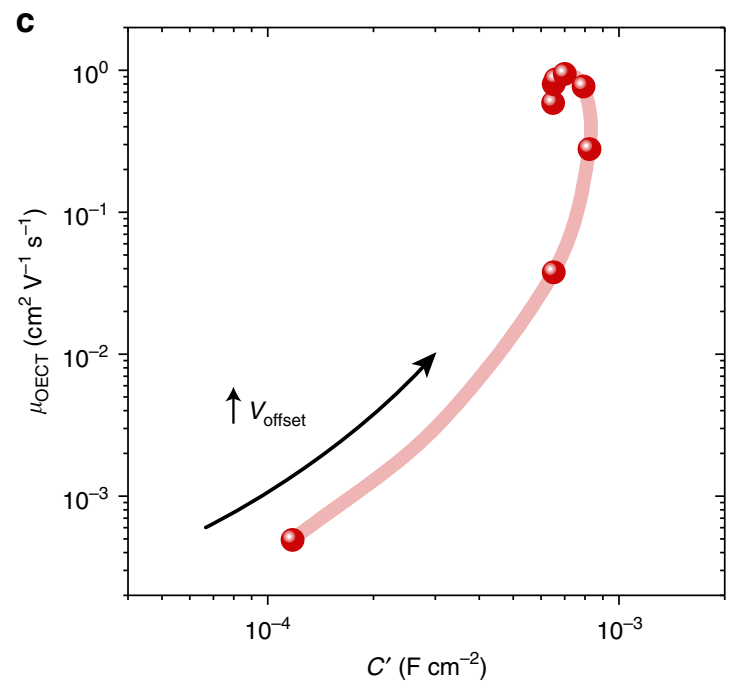

Fig. 3 Gate or offset bias trajectory for the p(g2T-TT). OECT mobility a and $C^{\prime}$ (capacitance per unit area) $\mathbf{b}$ as a function of bias ( $V_{\text {offset }}$ of working electrode for $C^{\prime},-V_{\mathrm{G}}$ for $\mu$ ). $\mathbf{c} \mu-C^{\prime}$ map of data from $\mathbf{a}, \mathbf{b}$ showing an example of a bias trajectory

$\boldsymbol{\mu}-\boldsymbol{C}^{\star}$ maps. By plotting the decoupled terms, $\boldsymbol{\mu}$ - $C^{\star}$ maps can be assembled to aide in materials understanding and synthetic design. On such a map, the highest $\mu C^{\star}$ product is at the top right (Fig. 2c), with lines of constant $\mu C^{\star}$ denoted as the dotted diagonal lines. PEDOT:PSS, labeled as (1) in Fig. $2 c$, has long been the top performing material. PTHS (2), a conjugated polyelectrolyte, was thought to be a promising candidate material due to its tethered ionic groups ${ }^{23}$, however the low $\mu C^{*}$ product suggests otherwise. By decoupling these terms, it is clear that while this material attains a $C^{*}$ value three times higher than that of PEDOT:PSS, its hole mobility is $\sim \times 1000$ lower. Thus, to further improve the performance of OECTs based on this material, focus should be placed on enhancing its mobility without hurting $C^{*}$. On the other hand, PEDOT:TOS (3), a vapor phase polymerized (VPP) PEDOT doped with tosylate (TOS) ions, shows a similarly high $C^{*}$ as PTHS, but maintains a mobility of $\sim 1 \mathrm{~cm}^{2} \mathrm{~V}^{-1} \mathrm{~s}^{-1}$. $\mathrm{p}(\mathrm{g} 2 \mathrm{~T}-\mathrm{TT})$ (4), the top performing OECT material in this data set, further pushes $C^{*}$ to $241 \mathrm{~F} \mathrm{~cm}^{-3}$, while still maintaining a high $\mu$. It is interesting to note that the only n-type material in this set, $\mathrm{p}(\mathrm{gNDI}-\mathrm{g} 2 \mathrm{~T})$, shows the highest $C^{*}$ value $\left(397 \mathrm{~F} \mathrm{~cm}^{-3}\right)$, and yet its mobility keeps it from attaining the high transconductance of its p-type counterparts ${ }^{22}$.
In this dataset, it is clear that $\mu$ varies over a larger range than $C^{\star}$. However, a number of trends are deduced from Fig. 2c: materials with comparable $\mu$ result in higher $\mu C^{*}$ products and thus higher transconductance due to a higher $C^{\star}$. Grouping materials, those possessing excess and bulky ionic or polyionic dopants, i.e. PEDOT doped with various polyanions ${ }^{24}$, show lower $C^{\star}\left(<100 \mathrm{~F} \mathrm{~cm}^{-3}\right)$ compared to the rest of the materials. These materials may allow for efficient swelling and faster ion drift and diffusion, however, the "dead volume" from the excess, electrically insulating phase lowers $C^{\star}$. Strategies for enhancing $C^{\star}$ are thus important, but doing so while maintaining high mobilities can present a tradeoff that must be deftly navigated ${ }^{19}$.

Trajectories. Nevertheless, distilling a material to a single point in $\mu-C^{\star}$ space can be misleading. For example, while often a mobility value is assigned to an organic electronic material, it is well known that mobility depends on charge density ${ }^{25}$, and can be anisotropic, depending on morphology 26,27 . Similarly, it has been observed in electrolyte gated organic transistors that as charge density is swept through a broad range (by sweeping the applied gate voltage), both mobility and the observed capacitance can 

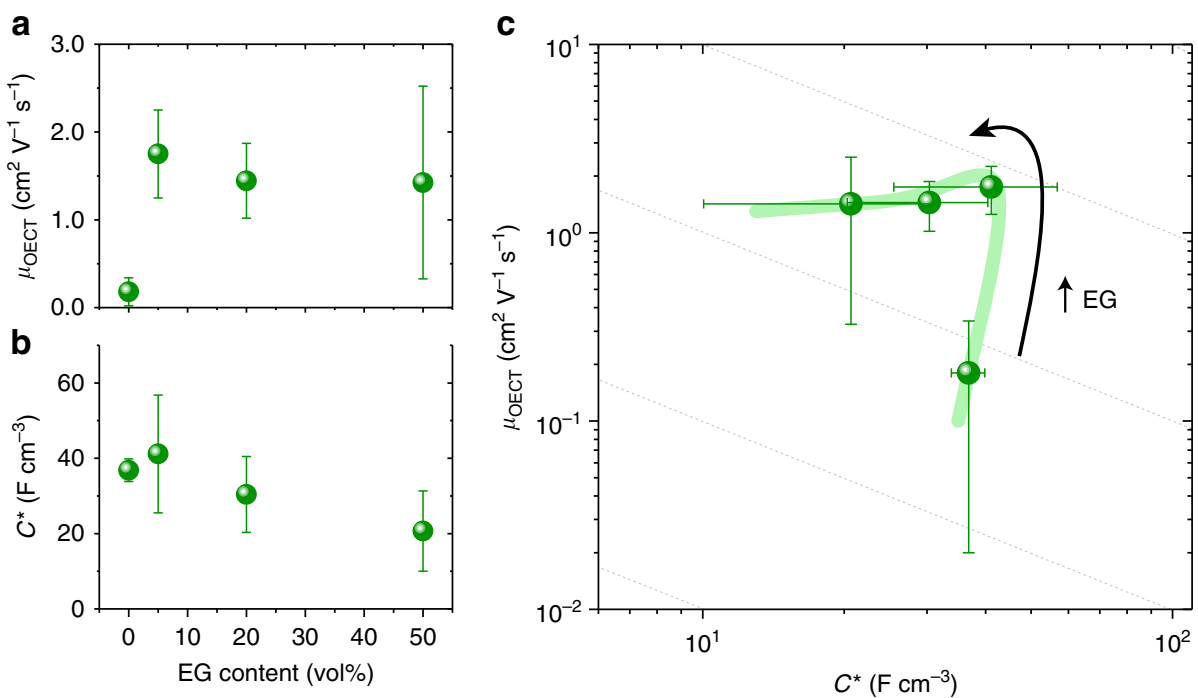

Fig. 4 Formulation/morphology trajectory for PEDOT:PSS. OECT mobility $\mathbf{a}$ and $C^{\star} \mathbf{b}$ as a function of ethylene glycol (EG) concentration. $\mathbf{c} \mu$ - $C^{\star}$ map of data from $\mathbf{a}, \mathbf{b}$ showing an example of a trajectory corresponding to variations in PEDOT:PSS formulation, and therefore the film morphology/microstructure. Dotted gray lines denote constant $\mu C^{\star}$ product. Samples are the same as those from ref. ${ }^{19}$. Error bars represent standard deviations from measurements of four transistors

vary significantly, often passing through a peak value 28,29 . For mobility, this may be attributed to band filling or in some cases, contact effects; for capacitance, the cause is potentially related to barriers for ionic/electronic charge injection. It should be carefully noted that the derivation of Eq. (1) (ideal OECT operation) assumes no such barriers. Thus, care must be taken in data interpretation when operating in regimes of significant voltage dependent capacitance, as noted below. Voltage dependence of $\mu$ and $C^{\star}$ is shown in Fig. 3 for an accumulation mode glycolated polymer, $\mathrm{p}(\mathrm{g} 2 \mathrm{~T}-\mathrm{TT})$. The electronic mobility, determined from an OECT, increases more than three orders of magnitude, with a peak value at $0.3 \mathrm{~V}$ (Fig. 3a). When biased over the same range, a drastic increase in capacitance starting at $V_{\text {offset }} \sim-0.1 \mathrm{~V}$ is noted, roughly saturating in the $0-0.6 \mathrm{~V}$ range (Fig. $3 \mathrm{~b}$ ). Such biasdependent functions can be represented in $\mu$-C space as in Fig. $3 \mathrm{c}$ - note that $C^{\prime}$ is reported rather than $C^{*}$ since the degree of uniformity of ion penetration through the bulk is unknown at low $V_{\mathrm{G}}$. As mentioned, the applicability of the figure of merit does not necessarily hold over the entire range. The highest transconductance for this device is achieved at the top right of this trajectory which would correspond to the largest $\mu C^{*}$ product; in this case at $V_{\text {offset }} \sim 0.25 \mathrm{~V}$, where proper OECT scaling is observed. All reported single $\mu$ - $C^{\star}$ values (Fig. 2) are at peak transconductance in saturation regime, which, in some cases, is the peak transconductance achieved before sample degradation or instability.

We can envisage other trajectories that can inform a processing or formulation arc maximizing mixed conductivity. An example of this type of trajectory is one where additives or solvent treatments are applied in order to tune the microstructure of the material, and therefore its properties. For instance, ethylene glycol (EG) is a co-solvent commonly used to enhance electronic conductivity of PEDOT:PSS. Recently, we explored how this additive simultaneously affects ion transport ${ }^{19}, 30$. This same formulation set (with EG) can be examined with respect to $\mu$ and $C^{\star}$ (Fig. $4 \mathrm{a}, \mathrm{b}$ ). The $\mu$-C $C^{\star}$ mapping of this trajectory (Fig. $4 \mathrm{c}$ ) is in further agreement with the results in ref. ${ }^{19}$, showing that a peak transconductance occurs at an intermediate EG concentration.

\section{Discussion}

Aside from such processing conditions, the peak $\mu C^{\star}$ product of a material can depend on other factors. Temperature, for example, is an obvious variable. Well controlled experiments with liquid (aqueous) electrolytes are, however, complicated when fine temperature control is desired, limiting the range of accessible temperatures as the morphology of the channel material and the phase and ion concentration of the electrolyte can simultaneously change. A resulting implication is that the $\mu C^{*}$ product does not depend solely on the dry material properties of the channel. It should also be a system property encompassing the nature of the electrolyte. The size, charge, and nature of the (solvated) ions would have differing ion mobilities ${ }^{31}$, and different interactions with the solid state film; in some cases a large ion may not be able to access the electroactive volume, which may limit $C^{*}$. In the extreme case, excessively large ions would not be able to penetrate the channel, resulting in a true "field effect" condition, where accumulation would be limited to double layer formation at the channel-electrolyte interface. The solvent of the electrolyte (in the case of liquid electrolytes) could also interact and modify the channel morphology depending on the nature of both polymer and solvent-i.e., hydration and swelling-which could affect both mobility and $C^{*}$.

It is noteworthy that while the $\mu C^{\star}$ product governs the steady state characteristics of an OECT, it holds little relevance to the transient behavior for materials that show true OECT operation. In fact, $g_{\mathrm{m}}$ does not include information directly associated with the time scale of (de)charging of the film. The response time of the OECT will be a function of how fast the ions penetrate inside the polymer and the electrons/holes are extracted at the source/ drain contacts. While much is known about electronic charge transport, the drift of ions inside the film along multiple directions and how this contributes to the switching speed of the device are challenging to quantify. However, for the material to be volumetrically doped/dedoped within the channel of an OECT on application-relevant time scales, we postulate that there should not be a significant barrier for ion injection and that the mobility of ions must be sufficiently high to allow for complete (de) charging of the (semi)conductor channel. While materials such as PEDOT:PSS show an agreement between the device response time and the $R C$ charging time of the transistor channel ${ }^{15}$, this is likely not true for all materials herein. Nevertheless, even the polymers for which ion transport is considered quite slow or at least orders of magnitude slower than PEDOT:PSS (e.g., p(g2T- 
TT), the top performer) are able to show OECT behavior and fit within the steady state $\mu C^{\star}$ framework.

While ultimately, the $\mu C^{\star}$ product can depend on a number of factors, it is a powerful tool to benchmark materials and can be used to directly compare materials being used as the active channel in OECTs. We have shown that of nearly a dozen reported materials for OECTs, representing the synthetic and materials development work of six academic groups, the magnitude of the $\mu C^{*}$ figure of merit varies over three orders of magnitude. The deconvolution of electronic mobility and the volume density of bulk charge storage (indicative of ion penetration/ transport) aides in materials design, and can thus be used to discuss organic mixed conductors in a common framework. While directly applicable for studying OECT performance from the transconductance perspective, relative materials benchmarking can be a powerful tool to compare organic mixed conductors of interest in other application areas.

\section{Methods}

OECT characterization. Materials and devices were prepared, as previously reported ${ }^{19,22-24,32-35}$. They were all tested as noted in Fig. 1, with a Ag/AgCl electrode (Warner Instruments) immersed in $0.1 \mathrm{M} \mathrm{NaCl}$ in deionized water. OECT characterization was performed on either Keithley 2400 source measure units (SMU) or a National Instruments PXIe system (equipped with NI-PXI-4071 digital multimeters, NI-PXI-4143 SMU and a NI-PXI-6289 DAQ), using custom LabView software. Mobility $\left(\mu_{\mathrm{OECT}}\right)$ was determined on the OECT channels the same or comparable to those used to measure the current-voltage characteristics and to extract the transconductance of the OECTs (i.e., same film casting consitions, comparable dimentions, and same effective gate biasing). Measurements were performed by using the constant gate current method, where drain current transients $\left(\mathrm{d} I_{\mathrm{D}} / \mathrm{d} t\right)$ are extracted for different constant gate currents, $I_{\mathrm{G}}$, at a particular drain bias, $V_{\mathrm{D}}$. The hole or electron transit time can then be calculated from $\mathrm{d} I_{\mathrm{D}} / \mathrm{d} t=-I_{\mathrm{G}} / \tau_{e}{ }^{17,20}$. Using the same relation, a frequency dependent approach can be employed to extract the hole/electron transit time. A constant drain bias and a sinusoidal voltage signal $\left(\Delta V_{\mathrm{G}}=10-20 \mathrm{mV}\right)$ at a gate voltage offset $\left(V_{\mathrm{G}, \text { offset }}\right)$ are applied depending on the biasing conditions of peak transconductance (similar to EIS experiments). The resulting gate current and drain current sinusoids are extracted to determine both amplitudes and phase shifts. The frequency domain relation derived from the time domain analysis is: $\Delta I_{\mathrm{G}}(f)=2 \pi f \tau_{e} \Delta I_{\mathrm{D}}$. Thus, matching the gate current and drain current derived impedance yields $\tau_{e}$, as detailed in ref. ${ }^{21}$. Using the hole/electron transit time in the channel $\left(\tau_{e}\right)$, the channel length, and the applied drain bias, $\mu_{\mathrm{OECT}}$ can be estimated.

Electrochemical impedance spectroscopy. Electrochemical impedance spectroscopy (EIS) for determination of capacitance was measured on polymer coated electrodes using either a Metrohm Autolab PGSTAT128N or Gamry Reference 600 from $20 \mathrm{kHz}$ to $1 \mathrm{~Hz}$. The impedance spectra of the channel of the OECT or a micron-scale film coated on gold electrodes $\left(3.48 \mathrm{E}-3 \mathrm{~cm}^{2}\right.$ in area) were measured in $0.1 \mathrm{M} \mathrm{NaCl}$ aqueous solution, using a standard $\mathrm{Ag} / \mathrm{AgCl}$ as the reference electrode and a Pt mesh as the counter electrode. The measurements were performed at a DC offset potential which enables the maximum achievable doping for the material and an AC amplitude of $10 \mathrm{mV}$. Once the spectra were recorded, they were fit to an equivalent circuit using native tool software (Metrohm Autolab NOVA or Gamry software) or MATLAB. The simplest circuit with a pure capacitor was used (i.e., $R_{\mathrm{s}} C$ ), or Randle's circuit, $R_{\mathrm{s}}\left(R_{\mathrm{p}} \| C\right)$, and resulted in good fit quality as shown in Supplementary Fig. 1 . The capacitance values that are extracted were normalized by the measured film volume or plotted as a function of volume and then fit to a line to determine volumetric capacitance $\left(C^{*}\right)$. Thickness of films was measured in the dry state with a Bruker Dektac profilometer.

Data availability. The data that support the findings of this study are available within the article, its Supplementary Information or from the authors.

Received: 10 July 2017 Accepted: 17 October 2017

Published online: 24 November 2017

\section{References}

1. Riess, I. Mixed ionic-electronic conductors-material properties and applications. Solid State Ion. 157, 1-17 (2003).

2. Liu, T. et al. Polyaniline and polypyrrole pseudocapacitor electrodes with excellent cycling stability. Nano Lett. 14, 2522-2527 (2014).
3. Malti, A. et al. An organic mixed ion-electron conductor for power electronics. Adv. Sci. 3, 1500305 (2016).

4. Amanchukwu, C. V. et al. Evaluation and stability of PEDOT polymer electrodes for Li-O2 batteries. J. Phys. Chem. Lett. 7, 3770-3775 (2016)

5. Winther-Jensen, B., Winther-Jensen, O., Forsyth, M. \& MacFarlane, D. R. High rates of oxygen reduction over a vapor phase-polymerized PEDOT electrode. Science 321, 671-674 (2008).

6. Mikhnenko, O. V., Collins, S. D. \& Nguyen, T.-Q. Rectifying electrical noise with an ionic-organic ratchet. Adv. Mater. 27, 2007-2012 (2015).

7. Smela, E. Conjugated polymer actuators for biomedical applications. $A d v$. Mater. 15, 481-494 (2003).

8. Argun, A. A. et al. Multicolored electrochromism in polymers: structures and devices. Chem. Mater. 16, 4401-4412 (2004).

9. Persson, K. M. et al. Matrix addressing of an electronic surface switch based on a conjugated polyelectrolyte for cell sorting. Adv. Funct. Mater. 25, 7056-7063 (2015).

10. Lin, P. \& Yan, F. Organic thin-film transistors for chemical and biological sensing. Adv. Mater. 24, 34-51 (2012).

11. Khodagholy, D. et al. Organic electronics for high-resolution electrocorticography of the human brain. Sci. Adv. 2, e1601027 (2016).

12. Nilsson, D., Robinson, N., Berggren, M. \& Forchheimer, R. Electrochemical logic circuits. Adv. Mater. 17, 353-358 (2005).

13. Gkoupidenis, P., Schaefer, N., Strakosas, X., Fairfield, J. A. \& Malliaras, G. G. Synaptic plasticity functions in an organic electrochemical transistor. Appl. Phys. Lett. 107, 263302 (2015).

14. Lin, P., Yan, F., Yu, J., Chan, H. L. W. \& Yang, M. The application of organic electrochemical transistors in cell-based biosensors. Adv. Mater. 22, 3655-3660 (2010).

15. Rivnay, J. et al. High-performance transistors for bioelectronics through tuning of channel thickness. Sci. Adv. 1, e1400251 (2015).

16. Khodagholy, D. et al. In vivo recordings of brain activity using organic transistors. Nat. Commun. 4, 1575 (2013).

17. Khodagholy, D. et al. High transconductance organic electrochemical transistors. Nat. Commun. 4, 2133 (2013).

18. Wang, H., Ail, U., Gabrielsson, R., Berggren, M. \& Crispin, X. Ionic seebeck effect in conducting polymers. Adv. Energy Mater. 5, 1500044 (2015).

19. Rivnay, J. et al. Structural control of mixed ionic and electronic transport in conducting polymers. Nat. Commun. 7, 11287 (2016).

20. Bernards, D. A. \& Malliaras, G. G. Steady-state and transient behavior of organic electrochemical transistors. Adv. Funct. Mater. 17, 3538-3544 (2007).

21. Rivnay, J. et al. Organic electrochemical transistors for cell-based impedance sensing. Appl. Phys. Lett. 106, 043301 (2015).

22. Nielsen, C. B. et al. Molecular design of semiconducting polymers for high performance organic electrochemical transistors. J. Am. Chem. Soc. 138, 10252-10259 (2016).

23. Inal, S. et al. A high transconductance accumulation mode electrochemical transistor. Adv. Mater. 26, 7450-7455 (2014).

24. Inal, S. et al. Organic electrochemical transistors based on PEDOT with different anionic polyelectrolyte dopants. J. Polym. Sci. B Polym. Phys. 54, 147-151 (2016).

25. Bouhassoune, M., Mensfoort, S. L. Mv, Bobbert, P. A. \& Coehoorn, R. Carrierdensity and field-dependent charge-carrier mobility in organic semiconductors with correlated Gaussian disorder. Org. Electron. 10, 437-445 (2009).

26. Kline, R. J. \& McGehee, M. D. Morphology and charge transport in conjugated polymers. J. Macromol. Sci. Polymer Rev. 46, 27-45 (2006).

27. Coropceanu, V. et al. Charge transport in organic semiconductors. Chem. Rev. 107, 926-952 (2007).

28. Ofer, D., Crooks, R. M. \& Wrighton, M. S. Potential dependence of the conductivity of highly oxidized polythiophenes, polypyrroles, and polyaniline: finite windows of high conductivity. J. Am. Chem. Soc. 112, 7869-7879 (1990).

29. Paulsen, B. D. \& Frisbie, C. D. Dependence of conductivity on charge density and electrochemical potential in polymer semiconductors gated with ionic liquids. J. Phys. Chem. C 116, 3132-3141 (2012).

30. Inal, S., Malliaras, G. G. \& Rivnay, J. Optical study of electrochromic moving fronts for the investigation of ion transport in conducting polymers. J. Mater. Chem. C 4, 3942-3947 (2016).

31. Stavrinidou, E. et al. Direct measurement of ion mobility in a conducting polymer. Adv. Mater. 25, 4488-4493 (2013).

32. Giovannitti, A. et al. Controlling the mode of operation of organic transistors through side-chain engineering. Proc. Natl Acad. Sci. USA 113, 12017-12022 (2016).

33. Winther-Jensen, B. \& West, K. Vapor-phase polymerization of 3, 4ethylenedioxythiophene: a route to highly conducting polymer surface layers. Macromolecules 37, 4538-4543 (2004).

34. Harman, D. G. et al. Poly(3,4-ethylenedioxythiophene):dextran sulfate (PEDOT:DS) - A highly processable conductive organic biopolymer. Acta. Biomater. 14, 33-42 (2015).

35. Giovannitti, A. et al. N-type organic electrochemical transistors with stability in water. Nat. Commun. 7, 13066 (2016). 


\section{Acknowledgements}

We are thankful to the following chemists for providing materials and support: Alexander Giovannitti and Iain McCulloch (Imperial/KAUST) for $\mathrm{p}(\mathrm{g} 2 \mathrm{~T}-\mathrm{TT}), \mathrm{p}(\mathrm{g} 2 \mathrm{~T}-\mathrm{T})$, $\mathrm{p}$ (gBDT-g2T), and p(gNDI-g2T); Mukundan Thelakkat (Bayreuth) for PTHS; Gordon Wallace and Paul Molino (Wollongong) for PEDOT:DS; and George Hadziioannou (Bordeaux) for PEDOT:PSTFSI and PEDOT:PMATFSI. The authors would like to thank Ilke Uguz and Mary Donahue for their assistance in device fabrication.

\section{Author contributions}

J.R. conceived and oversaw the research. S.I. and J.R. characterized electrochemical transistors, performed impedance spectroscopy measurements, and analyzed results. All authors discussed analysis and results. J.R. and S.I. wrote the manuscript. All authors provided input during manuscript preparation and revisions.

\section{Additional information}

Supplementary Information accompanies this paper at doi:10.1038/s41467-017-01812-w.

Competing interests: The authors declare no competing financial interests.
Reprints and permission information is available online at http://npg.nature.com/ reprintsandpermissions/

Publisher's note: Springer Nature remains neutral with regard to jurisdictional claims in published maps and institutional affiliations.

(c) (i) Open Access This article is licensed under a Creative Commons Attribution 4.0 International License, which permits use, sharing, adaptation, distribution and reproduction in any medium or format, as long as you give appropriate credit to the original author(s) and the source, provide a link to the Creative Commons license, and indicate if changes were made. The images or other third party material in this article are included in the article's Creative Commons license, unless indicated otherwise in a credit line to the material. If material is not included in the article's Creative Commons license and your intended use is not permitted by statutory regulation or exceeds the permitted use, you will need to obtain permission directly from the copyright holder. To view a copy of this license, visit http://creativecommons.org/ licenses/by/4.0/.

(C) The Author(s) 2017 